\title{
Dynamic Security Margin Estimation using Artificial Neural Networks
}

\author{
A. Sittithumwat Kevin Tomsovic \\ School of Electric Engineering and Computer Science \\ Washington State University \\ Pullman, WA 99164 USA
}

\section{INTRODUCTION}

Power System security is the ability of system to withstand sudden disturbances with minimum disruption to its quality of service. Examples of such disturbances are electric short circuit, change of transmission system configurations due to faults, loss of system components, line switching actions, or sudden load incrcasc. For the proper planning and operation, it means that after the disturbance occur, the power system will have to meet two requirements: (1) surviving the ensuing transient and move into an acceptable steady-state condition, and (2) in this new steady-state condition all components are operating within established limits [1]. The analysis used for the first requirement is so called transient analysis or Dynamic Security Assessment (DSA) while that for the second condition is known as static security assessment (SSA). DSA is the topic of concern for this paper.

Typically, the SSA is performed first and then the dynamic security assessment. The static security analysis evaluates the post contingent steady state of the system neglecting the transient behavior and any other time-dependent variations. On the other hand, the dynamic security analysis evaluates the time-dependent transition from the pre-contingent to the post-contingent state, specifically, the stability of the system both from small and large disturbances. Several techniques are now available to perform quickly and reliably only for SSA, and most Energy Management Systems (EMS) can perform this type of study. DSA methods are not fully operational and are currently being tested and evaluated.

Two dynamics problems, i. e., transient stability and voltage collapse, should be considered when performing a dynamic security study. Transient stability assessment is a major concern in the DSA for multi-machine power systems. Particularly, a fault on the system or a loss of a large generator can give rise to large electromechanical oscillations between generating units, which might lead to loss of synchronism in the system. For the voltage stability problem, it is associated with the increased loading of long transmission lines and insufficient local reactive power supply. These types of phenomena are characterized by a voltage drop gradual at first, and then collapse. The time interval of the slow voltage decay phase typically is in between 1 to 10 minutes, which is adequate for the operator to exercise the corrective action. This paper focuses on transient stability.

\section{BRIEF LITERATURE REVIEW}

Various methods are available for assessing the transient stability. Conventional transient stability analysis approaches usually use very extensive mathematical model of the system that are computational extensive and time-consuming. The training based approaches use off-line generation of pattern sets to train the assessors. If conventional techniques are used to analyze dynamic security studies, they may use excessive time on the EMS computers at the cost of other computations and functions that need to be performed by the EMS. Therefore, these are undesirable for on-line purposes. There is a need for fast and accurate tools to evaluate dynamic security. These problems can be handled using non-analytical approaches. Among these techniques, artificial neural networks (ANNs) are one of the most promising methods [2] because ANNs have excellent generalization capabilities, superior noise rejection, and fast execution (most of the calculations occur during the initial off-line training).

Over the past ten years or so, a number of approaches using artificial neural networks have been proposed as alternative methods for dynamic security assessment in power system operations. 
Sobajic and Pao have proposed a technique using a multi-layered network to predict the critical clearing time (CCT) for a given system disturbance [3]. In this paper, the authors have shown that the neural networks can generalize to previously unencountered system topologies and load levels, and correctly estimate CCTs. In a follow up, they also presented a combined unsupervised and supervised learning algorithm to solve the same problem [4]. The input data was pre-processed using an unsupervised learning system in order to enhance the accuracy of the supervised learning algorithm.

El-Sharkawi et al. exploited a multi-layer perceptron to predict the dynamic stability status of power system [5]. A layered perceptron was trained to learn the mapping between varying system operating state, active and reactive power injections in some system buses and the corresponding dynamic security status. Kumar et al. [6] discussed implementing the $\mathrm{NN}$ for the dynamic security assessment in a large system, and proposed a hybrid expert system/neural network approach, which utilize the knowledge of system operators while training neural works. In Mansour et al. [7], a neuron network approach to provide contingency screening and ranking in dynamic security was proposed. The B. C. Hydro and Hydro Quebec systems were used to test the neuron network. The static and dynamic features were applied as inputs, and the selected outputs were energy margin and maximum swing angle.

\section{PROBLEM FORMULATION}

Power system security assessment can be divided in to two categories, i. e., classification and security limit determination. Classification includes determining whether the system is secure or insecure under all pre-specified contingencies. Classification does not in itself indicate the distance from the operating condition to the insecure conditions. Security limit determination, on the other hand, involves finding this distance. Safe operating levels based on various system conditions are given in terms of a critical system opcrating parameter, e. g., loading of a certain power plant, the power flow at critical transmission interface, the voltage at given bus, and so on.
Reliability standards require that the system should be operated with sufficient margins to withstand any single contingency without experiencing overloads, low voltages, and sustained frequency dip. For most utilities, the margin for each study case is used to produce a two-dimensional graph called a nomogram, of which the two axes correspond to two critical parameters, and all other critical parameters are set to selected values within a typical operating range. The drawbacks include inaccurate boundary representation and little flexibility because there are approximations made in developing nomogram, i. e., linear interpolation between points and insufficient information contained in critical parameters. These disadvantages can be improved using neural networks to estimate the security margins.

In this paper, the power system security limit in response to all predefined contingencies is investigated for a given pre-contingency steady state of the system. The loading is varied to determine the security margin regarding to frequency deviation security. The allowable margins and associate reliability criteria are based on the regional council (WSCC) guidelines [8].

Criteria for dynamic security assessment typically include:

- Inertial stability criteria. This concerns mainly the evolution of relative machine angles and frequencies.

- Voltage excursions (dip or rise) beyond specified threshold level and duration. This includes separate voltage excursion threshold/duration pairs for voltage dip and voltage rise, and maximum/minimum instantaneous excursion threshold.

- Relay margin criteria. These are defined for pre-disturbance and post-disturbance conditions. If relay margin is violated for more than a maximum specified time after the disturbance, it is identified as insecure.

- Minimum damping criteria. For a designated list of contingencies, if the postdisturbance system exhibits oscillations, they must be positively damped (decreasing in amplitude). 
Identifying the specific set of security constraints to be introduced for the dynamic security studies is based on experience, knowledge of the system, and judgment of the planning and operations engineers.

\subsection{Proposed Approach}

The ANN used in this study is the feed-forward multi-layer structure. Our focus here is on estimation of the loading margin ( $\mathrm{P}$ margin) regarding to transient frequency criteria $(59.6 \mathrm{~Hz}-$ 6 cycles). Note that the amount of additional load of a particular operating point that would violate the frequency criteria is called the loading margin to frequency deviation. The active and reactive power flow of all lines is used as the features in order to describe a system state. The ANN is trained using the data of the off-line operational studies.

\section{Data Generation}

To determine the transient stability transfer limits or loading margins, a load-flow software package (IPFLOW) must first be executed for a given topology to have a satisfactory steady-state case. This steady-state case is then applied to initialize the network for a transient-stability simulation program (ETMSP). When the simulation is finished, reliability criteria are exercised with the extract results from ETMSP, and the next step will be determined, and the load-flow software inputs will be modified accordingly and then the process will be reiterated. This is repeated till the highest stable transfer level for an interface is found. In general, to find the security limit, the process must be repeated for different contingency types and location until the most constraining (i. e., lowest) transfer limit has been identified. Note that it is impossible to pre-study all possible contingencies because the assessment processes are very timeconsuming, and the analysis of every degraded topology is a problem of combinatorial dimensions.

\section{Neuron Network Design}

A feed-forward ANN based on LevenbergMarquardt and Bayesian Regulation backpropagation is applied [9]. It includes three layers: one input, one hidden, and one output. The number of relevant features needed to describe the domain identifies the size of input. Similarly, the size of the output layer is determined by the items desired to be estimated. There is no exact criterion for the number of hidden layers nor the number of neurons per hidden layer. Too many neurons can lead to memorization of the training data with the danger of losing its ability to generalize and predict. Data or training sets have to be representative of the different states of the power system because NNs are good in interpolation but not extrapolation. This means that they need to contain the complete pattern space.

\section{Feature Selection}

This stage can be considered as a pre-processing step. This is an extremely important step, as selected features should characterize properly a variety of power system operating conditions. Generally, the dimension of the pattern vector is very large. The process of finding the most significant variables, eliminating redundancy and reducing the dimension of the pattern vector is called feature selection. Different methods based on statistical approaches are available for feature extraction (i. e., reducing the dimension of the input data vector). Here, parameters that may be applied to describe a system state are as follows:

- The voltage magnitude and phase angle at each system bus

- The active and reactive power of each bus load

- The active and reactive power flow of all the lines

- The active and reactive power output of each generator plant

The selection and extraction process involves engineering judgment and statistical approaches. The statistical approaches applied in the study are correlation coefficients and principal components analysis (also called Karhunen-Loeve expansion) [9]. The correlation coefficients between the selected features and the computed security margin are firstly determined. Subsequently, principal components analysis can be exploited. The principal component analysis method determines 
the eigenvectors corresponding to the largest eigenvalues of the auto-correlation matrix of training vectors as its principal components. The reduced training vectors are selected in the direction of the most dominant eigenvectors. The ANN is then trained with this new set of reduced vectors. This extraction closely relates to the performance of a neural network and computation time since the less the number of features, the less samples are required.

\section{Splitting data for estimation and validation}

One of the simplest and most widely used means of avoiding overfitting that might happen when training NNs is to divide the training data into two sets: an estimation set and a validation set. The optimum ratio $r_{\text {opt }}$ that determines the split of the training data between estimation and validation sets is defined by

$$
r_{o p t}=1-\frac{\sqrt{2 W-1}-1}{2(W-1)}
$$

where $r_{\text {opt }}$ is the estimation portion, $W$ is the number of free parameters in the network with $N<W$, and $N$ is the size of the training set [10].

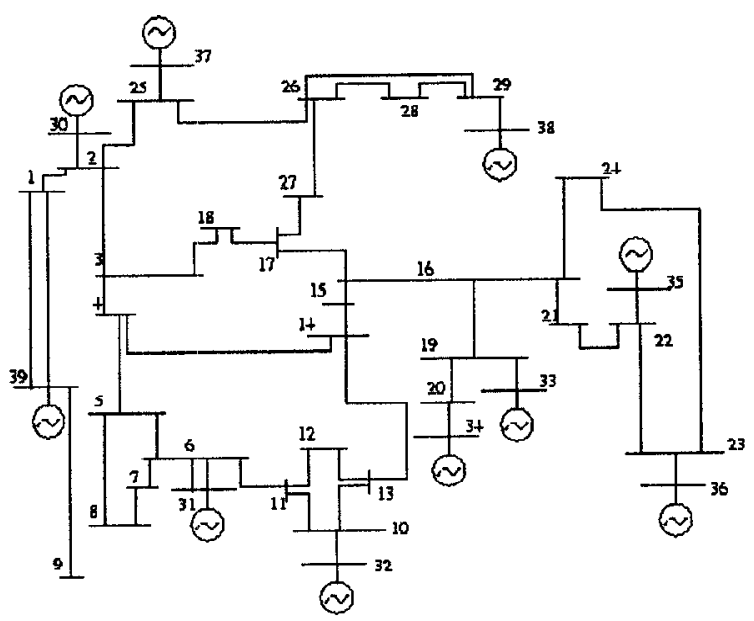

Figure 1 New England 39 Bus System

\section{NUMERICAL EXAMPLE}

To illustrate the proposed approach the New England 39 bus system is chosen. The system is divided into two zones, one load center with only load buses including buses 17, 18, and 27 with three tie lines 3-18, 16-17, and 26-27 as shown in Figure 1. The other contains all the other load and generation buses. The focus of study here is the power flow at the interface of this load center.

The $\mathrm{P}$ margin is decided by increasing the active power of the load center step by step over the base case until there is a violation of the reliability criteria (in this study, either frequency deviation or inadequate damping). The smallest step used is 25 MW, and the total load for the base case is 6000 MW. The entire data set consists of 983 samples, with $20 \%$ for testing and $30 \%$ for validation. The training and testing data obtain from transient stability studies using IPFLOW and ETMSP. Contingencies considered are the three-phase fault on each line. For each load level, therc arc 31 cases representing one base case and 30 (n-1) contingencies.

Initially, there are 96 total features, i.e., the active and reactive power flow of all the lines. These reduce to 31 features using principal components analysis. Here, statistical correlation coefficients were exploited to further reduce the dimension of the pattern vector to 11 elements. An ANN with 21 neurons in the single hidden layer was implemented using the Levenberg-Marquardt algorithm, and an ANN with 7 neurons of the single hidden layer was found to be adequate for Bayesian Regulation backpropagation.

From the results, shown in Table 1 and Figure 2, the performance of the ANN using Bayesian Regulation algorithm is more accurate than Levenberg-Marquardt algorithm. Further, since the Bayesian Regulation algorithm has fewer hidden neurons, training using this algorithm is faster in computation time. 


\section{CONCLUSION}

The ANN results obtained from the 39-bus system show that this technique is able to predict the security margin with a reasonable degree of accuracy. Prediction and generalization capabilities of these NNs provide the flexible mapping of input attributes to the single-valued space of security margin. Since ANNs have high computation rates, parallel distributed processing, fault tolerance, and adaptive capability, they are an excellent tool for on-line application in the areas in which data requirements and computational burden have rendered other approaches impractical for implementation.

Further efforts are needed to develop this approach into an efficient dynamic security assessment tool for large systems. These attempts by the authors include:

- Development of an automated procedure that generates a large database with each record comprising pre-disturbance operating parameters and the corresponding postdisturbance performance measure.

- Application of this approach to the WSCC system.

- Development of various ANNs for different operating conditions (restricting the domains of validity for each ANN)

\section{References}

[1] IEEE Committee Report, "Dynamic Security Assessment Practices in North America," IEEE Transactions on Power Systems, Vol. 3, No. 3, August 1988, pp. 1310-1321.

[2] Moechtar, M., Cheng T. C., and $\mathrm{Hu}$ L., "Transient Stability of Power System - A Survey," WESCON conference record Proceedings of 1995, San Francisco, California, November 7-9, 1995, pp. 166-171.
[3] Sobajic, D. and Pao, Y., "Artificial Neural Net Based Dynamic Security Assessment for Electric Power Systems," IEEE Transactions on Power Systems, Vol. 4, No. 1, February 1989, pp. 220-228.

[4] Pao, Y. and Sobajic, D. J., "Combined Use of Unsupervised and Supervised Learning for Dynamic Security Assessment," IEEE Transactions on Power Systems, Vol. 7, No. 2, February 1989, pp. 878-884.

[5] El-Sharkawi, M. A., Marks, R. J. II, Aggoune, M. E., Park, D. C., Damborg, M. J., and Atlas, L. E., "Dynamic Security Assessment of Power Systems Using Back Error Propagation Artificial Neuron Networks," Proceedings of the $2^{\text {nd }}$ Symposium on Expert System Applications to Power Systems, Seattle, WA, July 1989.

[6] Kumar, R., Ipahchi, A., Brandwajan, V., ElSharkawi, M. A. and Cauley, G., "Neuron Networks for Dynamic Security Assessment of Large Scale Power Systems: Requirements Overview," Proceedings of $I^{\text {st }}$ International Forum on Applications of Neuron Networks to Power Systems, Seatlle, WA, July 1991, pp. 6571.

[7] ManSour, Y., Chang, A. Y., Tamby, J., Vaahedi, E., Corns, B. R., and El-Sharkawi, M. A., "Large Scale Dynamic Security Screening and Ranking Using Neuron Networks," IEEE Transactions on Power Systems, Vol. 12, No. 2, May 1997, pp. 954-960.

[8] Western Systems Coordinating Council, Reliability Criteria, August 1998.

[9] Haykin, S., Neural Networks a Comprehensive Foundation, $2^{\text {nd }}$ Edition, Prentice Hall, New Jersey, 1999.

[10] Chen, L., Tomsovic, K., Bose A., and Stuart, R., "Estimating Reactive Margin for Determining Transfer Limits," IEEE Transactions on Power Systems, in press. 


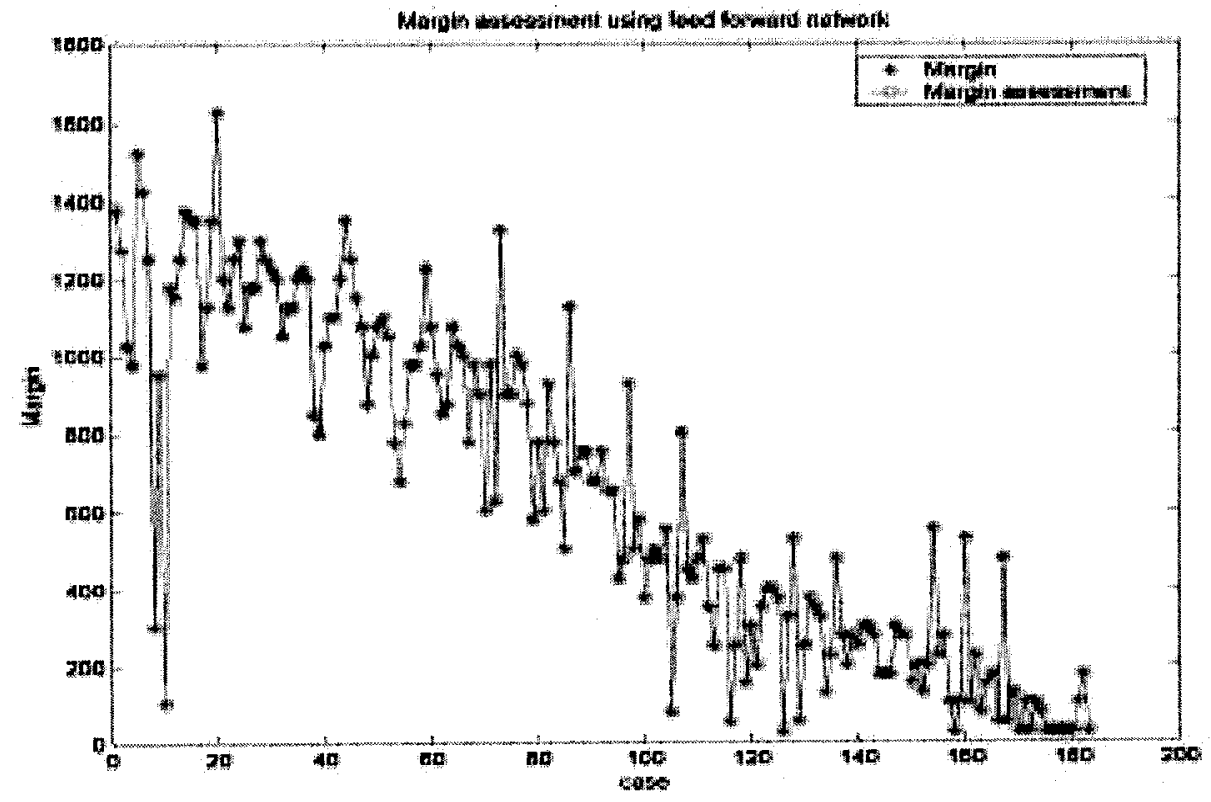

Figure 2 Performance on the 39 bus system

\begin{tabular}{|l|l|l|l|l|l|l|}
\hline $\begin{array}{c}\text { Training } \\
\text { Method }\end{array}$ & $\begin{array}{c}\text { Max error } \\
(\mathrm{MW} / \%)\end{array}$ & $\begin{array}{c}\text { Min error } \\
(\mathrm{MW} / \%)\end{array}$ & $\begin{array}{c}\text { Mean } \\
\text { error } \\
(\mathrm{MW} / \%)\end{array}$ & $\begin{array}{c}\text { Mean } \\
\text { Square error } \\
\left(\mathrm{MW}^{2}\right)\end{array}$ & $\begin{array}{c}\text { Standard } \\
\text { Deviation } \\
(\mathrm{MW})\end{array}$ & $\begin{array}{c}\text { Number of } \\
\text { hidden neurons }\end{array}$ \\
\hline $\begin{array}{l}\text { Levenberg- } \\
\text { Marquardt }\end{array}$ & $\begin{array}{l}8.1880 \\
3.8815 \%\end{array}$ & $\begin{array}{l}0.0024 \\
0.0005 \%\end{array}$ & $\begin{array}{l}0.4891 \\
0.1466 \%\end{array}$ & 0.6339 & 0.7956 & 21 \\
\hline $\begin{array}{l}\text { Bayesian } \\
\text { Regulation }\end{array}$ & 1.0752 & 0.0014 & 0.1460 & 0.0408 & 0.2023 & 7 \\
\hline
\end{tabular}

Table 1 Numerical Result of The 39 Bus System 